\section{Hand-arm vibration syndrome}

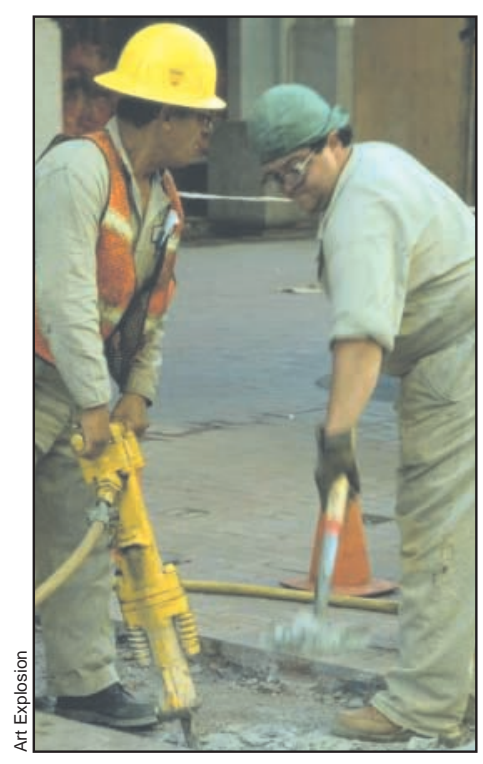

Background and epidemiology: Hand-arm vibration syndrome (HAVS) refers to a constellation of vascular, neurological and musculoskeletal signs and symptoms that may occur in workers who use handheld vibrating tools, in particular drills, grinders, electrical drills and saws, and jackhammers. Commonly afflicted occupational groups are shown in Box $1 .{ }^{1}$

The burden of HAVS is underreported, and the syndrome is often misdiagnosed as carpal tunnel syndrome. This is not surprising, since the 2 syndromes can coexist and HAVS can present with various degrees of sensory, vascular, muscular and osteoarticular involvement. It is important to distinguish the 2 syndromes, however, since some surgeries that are effective with carpal tunnel syndrome do not alleviate HAVS.

Typical clinical signs of HAVS are shown in Box 2. Symptoms do not present in a t predictable order, although in general the vascular changes have a longer latency period than the sensorineural deficits but are more commonly reported. ${ }^{2}$

The pathophysiologic features of HAVS are not well understood. Anatomic vascular changes occur with hypertrophy of the vessel wall and endothelial cell damage. The coldinduced vascular spasm is thought to be mediated by $\alpha-2$ adrenoreceptors in vessel walls. Pathologic changes have also been described in the digital tuft mechanoreceptors (Pacinian corpuscles) and among myelinated digital fibres. ${ }^{1}$

Physical characteristics of exposure that appear to play a role in the development of HAVS include the type of vibration (frequency, amplitude and direction), the vibratory force and impulse type, the cumulative hours of exposure, the intermittency of exposure, the state of tool maintenance and a cold environment. ${ }^{2}$ Biodynamic factors such as the grip force required to use the tool, the operator's control of the tool and the surface area of the tool handle, as well as factors such as the patient's medical history of injury, susceptibility and protective practices also play a role (www .ccohs.ca/oshanswers/phys_agents /vibration/vibration_effects.html \#_1_3). Symptoms typically do not appear until after 2000 hours of exposure to vibrations, ${ }^{2}$ although the latency period can vary considerably.

Clinical management: Diagnosis is based on a history of exposure to hand or arm vibration and sensorineural or vascular symptoms. Sensorineural tests in the clinic include electric current perception threshold, Semmes-Weinstein monofilaments, vibration perception threshold (tuning fork), 2-point discrimination and digital perception of small objects. Laboratory testing includes nerve conduction study and vibrometry testing. Several tests are used to assess the vascular component of HAVS. Plethysmography before and after cold provocation is the laboratory test accepted for evaluating vascular manifestations of HAVS by the Stockholm Hand-arm Committee. ${ }^{1}$ Additional tests include digital thermometry at baseline and after cold-water immersion and Doppler blood pressure and flow measurement. However, a recent review of the diagnostics of vascular symptoms concluded that taking a good patient history was the best method for diagnosing blanched fingers triggered by cold. ${ }^{3}$

Treatment includes maintaining core body temperature, avoiding exposure to cold and vibrating tools, job modification or a change of job, and splinting at night to treat neuropathies. Medications include calciumchannel blockers, pentoxyphylline to improve flexibility of red blood cells and drugs to reduce platelet deposition. Surgical intervention is neither successful nor warranted. ${ }^{1}$

Prevention: Preventive measures in the workplace are extremely

Box 1: Occupational groups at risk of hand-arm vibration syndrome

Farmers, forestry workers, stone drillers, stone cutters and chippers, quarry drillers, aircraft engine workers, sheet metal workers, polishers, grinders, moulders, cleaners, forklift drivers, engine testers, electric welders, riveters, dental technicians, orthopedists, sewing machine operators, chainsaw operators, construction workers, pedestal grinders, users of impact power tools, shipyard workers, railway workers

Box 2: Clinical signs of hand-arm vibration syndrome

- Blanched fingers

- Tenderness or pain and swelling of the fingers and forearm tissue

- Paresthesia or tingling in fingers

- Cold intolerance

- Weakness of the finger flexors or intrinsic muscles

- Loss of muscle control

- Reduced sensitivity to heat and cold

- Discoloration and trophic skin lesions of the fingers

- Loss of manipulative dexterity and finger coordination 
important. They include the use of ergonomic tools, antivibration padding and gloves to keep hands dry and warm, and reduction in the intensity of vibration to the hand through damping techniques, job rotations and scheduled rest periods. ${ }^{2}$ Efforts are underway to establish evidence-based limits to vibration exposure. Smoking cessation is also recommended, given the vasoconstrictive effects of cigarette smoking on the peripheral arteries.

\section{Erica Weir \\ CMA7}

Lina Lander

Doctor of Science candidate

Department of Environmental Health

Harvard School of Public Health

Boston, Mass.

\section{References}

1. Piligian G, Herbert R, Hearns M, Dropkin J, Landsbergis P, Cherniack $M$. Evaluation and management of chronic work-related musculoskeletal disorders of the distal upper extremity. Am 7 Ind Med 2000;37:75-93.

2. Friden J. Vibration damage to the hand: clinical presentation, prognosis and length and severity of vibration required. 7 Hand Surg [Br] 2001;26 (5):471-4.

3. Gosta G. Diagnostics of hand-arm system disorders in workers who use vibrating tools. Occup Environ Med 1997;54:90-5.

\section{Clinical Vistas}

\section{Alkaptonuria and photography: A patient's urine tells the story}

This picture of a patient 1 who had alkaptonuria (Fig. 1) was taken by my father, $\stackrel{\infty}{\sim}$ Dr. Ian Maxwell, in 1957 and was developed using the patient's own urine.

Alkaptonuria was the first described "inborn error of me" tabolism." This rare (< 1 per ○ 250000 births) hereditary reces-
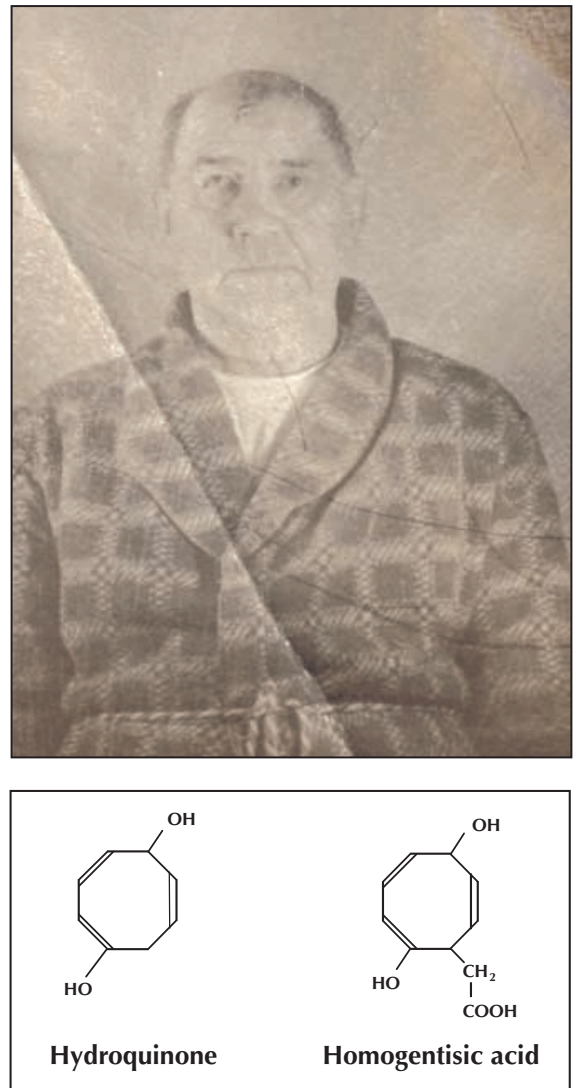

sive disorder is characterized by a triad of excretion of homogentisic acid in the urine, ochronosis (dark pigmentation of the connective tissues) and earlyonset arthritis. It results from the absence of an enzyme, homogentisic acid oxidase. Homogentisic acid is produced during the metabolism of phenylalanine and tyramine and, in the absence of the enzyme, accumulates in large quantities in the plasma and urine. In patients with alkaptonuria, a second enzyme, homogentisic acid polyphenoloxidase, present in mamallian tissue, catalyzes the oxidation of the accumulated homogentisic acid in vivo to an ochronotic pigment that has a high affinity for cartilage and connective tissues, forming fragile complexes. The clinical manifestations of the disease dense, coal-black pigmentation of costal, tracheal and laryngeal cartilages, blue-grey pigmentation of the ears and sclera, and extensive early degenerative arthritis of the spine and large joints (due to the breakdown of the cartilage-pigment complexes) - are the result of this pigment deposition. The excess homogentisic acid also appears in the urine, where it may undergo oxidation on exposure to atmospheric oxygen, particularly in an alkaline environment. This oxidation results in a characteristic gradual darkening of the urine downward from the surface on standing. ${ }^{1}$

To appreciate the relation of this to photography, we need a (somewhat simplified) explanation of the processes involved in the creation of a photographic image. The photographic emulsion consists of microscopic crystals of silver bromide embedded in a gelatin matrix. Photons striking the crystals dislodge electrons, resulting in the formation of metallic silver atoms and bromine. The latter is trapped by the gelatin matrix, leaving the silver atoms. At this stage the number of silver atoms is relatively small, creating what is termed a latent image. If this image is now "developed," by placing it in a solution containing a relatively strong reducing substance, the crystals in which the process of conversion to metallic silver has been initiated rapidly seize the electrons donated by the reducing substance, multiplying the original generation of metallic silver atoms by a factor of roughly 10000 . The developed image is now formed of crystals of metallic silver wherever light struck it, interspersed with unaltered crystals of silver bromide. The latter are then rendered soluble and washed out of the emulsion, 